ACTA SCIENTIFIC NEUROLOGY (ISSN: 2582-1121)

Volume 4 Issue 6 June 2021

\title{
Work Experiences as a Neurosurgeon in a Remote City
}

\section{Manish Kumar Dhiraj*}

General Surgery, Neurosurgery, Adya Neuro Care Hospital, Darbhanga, Bihar, India

*Corresponding Author: Manish Kumar Dhiraj, MS General Surgery, MCh

Neurosurgery, Adya Neuro Care Hospital, Darbhanga, Bihar, India.
Received: March 30, 2021

Published: May 17, 2021

(C) All rights are reserved by Manish Kumar

Dhiraj.
Being a Neurosurgeon I started working in the Darbhanga District of the Bihar province of India, which has one of the lowest per capita income in the Country. At that moment, hospitals in the town had no requisite infrastructure to perform brain surgeries. I was the first Neurosurgeon to start Neurosurgery in Darbhanga and Muzaffarpur Districts having 5.1 million population as per 2011 census data. It all started with construction of my own Adya Neuro Care Hospital with the help of financial support from The State Bank of India and HDFC Bank. The Hospital started functioning from December 2013. In the past 8 years I have managed thousands of patients which include almost 1,350 admitted patients of all age groups and more than 50,000 patients as outpatients. I have performed almost 200 major Brain and Spine Surgeries. Few of these patients were of challenging brain tumours such as mid para-sagittal meningioma, torcular meningioma, posterior fossa hemangioblastomas with hydrocephalous and gliomas. In the follow up of these tumour patients there is no recurrence till date, with overall follow up period of 6 years. I lost few patients of high-grade gliomas during follow up period, who discontinued follow up after a certain period of time. Other operated patients included extradural hematomas, acute and chronic subdural hematomas, intracerebral hematomas and contusions with mass effect, depressed fractures, brain abscesses and ventriculo-peritoneal shunts. Few patients whom I lost in post-operative period were above 60 years of age and had other comorbidities like uncontrolled hypertension and diabetes along with poor pre-operative Glasgow Coma Scale Score $^{1}$ (below 8/15). One of the few challenging Spine cases I had to operate upon was an elderly patient who had cauda equina syndrome in the form of bilateral foot drop along with the loss of control of bladder and bowel control. In two years of follow up he regained his bladder and bowel control and was able to walk with- out support. The overall recovery rate in admitted patients till date has been more than $95 \%$.

Hence in my last 8 years of work, I was able to:

- Create awareness in general population that Neurosurgery can be performed in remote areas and at low cost, (the average cost of major brain surgeries in my hospital is about Rs. $1,20,000 /-$, i.e. approximately $\$ 1,700$ including the cost of surgery, medicines and hospital stay), which is about 5 times less than the cost in big corporate Hospitals in India and much less compared to the cost incurred in the developed countries.

- I was able to convince the local medical fraternity that even complicated neurosurgical operations are possible in remote areas with very low mortality and low morbidity rates and good functional recovery of the patient.

- I was able to create an infrastructure which provided lowcost super specialty neurosurgical management to poor underserved sections of society who could not afford Medicare in costly corporate hospitals and the requisite infrastructure for their treatment did not exist in the Government Healthcare system locally.

During this entire tenure, I faced certain restraints which included creating funds for the development of infrastructure, interference and apathetic attitude of government regulatory authorities who created rules like Clinical Establishment Act favoring big Corporate Hospitals, convincing local medical fraternity who referred patients to the State or National capital and winning the trust of local general public that neurosurgery can be performed in smaller cities also. 
Still, I persisted and worked hard tirelessly against all odds to create an infrastructure which could serve the below poverty line sections of the society and taking Microscopic Neurosurgery to their doorstep. In future I wish to bring the advanced subspecialities of neurosurgery like vascular neurosurgery, endoscopic neurosurgery, functional and skull base neurosurgery and develop a culture of team work in small towns in order to serve the poor, underserved sections of the society [1].

\section{Acknowledgements}

I would like to acknowledge and thank, Prof. Dr. S N Rai, ex HOD Anesthesiology Dept. Darbhanga Medical College and Hospital (DMCH), Dr Sushil Kumar, Assistant Professor Anesthesiology Dept. DMCH, Dr Sajjan Kumar Mishra, Anesthesiology Dept. DMCH, Dr Swati Dhiraj, Adya Neuro Care Hospital, and Dr Rajiv Kumar, Adya Neuro Care Hospital, for supporting me throughout my endeavors and working as a cohesive team to take every possible care of the patients. Personally, I would like to thank Prof. Dr. R N Sharma ex HOD Pharmacology dept. Patna Medical College and Hospital for encouraging me to carry on despite facing hurdles.

\section{Bibliography}

1. Maconochie I and Ross M. "Head injury (moderate to severe)". BMJ Clinical Evidence (2007): 1210.

Volume 4 Issue 6 June 2021

\section{(C) All rights are reserved by Manish Kumar Dhiraj.}

